\title{
Editorial: Nanochemical Concepts for a Sustainable Energy Supply
}

\author{
D. S. $\mathrm{Su}^{\mathrm{a}}, \mathrm{A}$. Thomas $^{\mathrm{b}}$ \\ ${ }^{a}$ Fritz Haber Institute of the Max Planck Society, Faradayweg 4-6, 14195 Berlin (Germany. \\ ${ }^{\mathrm{b}}$ Technische Universität Berlin, Englische Straße 20, 10587 Berlin (Germany)
}

Published Online: 12 Feb 2010, published in print: 22 Feb 2010

The technical and industrial world as we know it is currently changing. This is caused by a number of serious problems, especially developing societies' need for energy. At present the world energy consumption is based for nearly $90 \%$ on fossil sources (i.e., coal, oil, natural gas), depleting these fossil sources and making them scarce and expensive. This is hard to compensate for developed economies, but bearing the energy needs of emerging countries in mind, staying fossil is not a sustainable option. A simple improvement of current knowhow and principles is thus no longer adequate for our future energy supply. But even assuming infinite fossil resources, unrestricted growth is not an option, as the industrializationbased $\mathrm{CO}_{2}$ loading of the atmosphere has already reached a level at which the impact on climate and living conditions are turning serious. These problems are now even generally perceived as the grand challenge for today's society, and the scientific community might want to accept the duty of showing possibilities and opportunities out of this "dinosaur trap."

To explore the potential role of basic science in this context, the project house "EnerChem" was founded in 2005, more specifically with the aim to find "Nanochemical Concepts for a Sustainable Energy Supply."

EnerChem was organized as a "virtual" institution between five Max Planck Institutes (MPIs), namely the MPI of Colloids and Interfaces, with the initiator and spokesman of the association, Markus Antonietti; the MPI for Polymer Research with Klaus Müllen; the MPI for Coal Research with Ferdi Schüth; the MPI for Solid State Research with Joachim Maier; and the Fritz Haber Institute of the Max Planck Society with Robert Schlögl.

Indeed, already promoting communication and cooperation between these related and similarly structured five Max Planck Institutes was a challenging task, because areas such as nanotechnology, catalysis, polymer chemistry, solid state chemistry, and electrochemistry had to be integrated under the transversal task of energy-related problems. It turned out that a common denominator, the chemistry of functional materials, worked to generate sustainable solutions for various alternative pathways towards the generation, conversion, storage,saving, and supply of energy.

After five years of EnerChem it can be now stated that especially because of this cooperation between scientists from different institutions and disciplines, a fruitful impact could be made by this highly targeted oriented approach. Indeed, materials ranging from organic molecules, polymers, and carbons to inorganic solid state materials have been discovered and analyzed for different purposes, from applications in fuel cells and lithium-ion batteries to the production and storage of hydrogen, and for catalytic schemes based on sustainable materials.

This EnerChem Special Issue contains some results of this fiveyear cooperation, while more than 150 papers have been pub- lished elsewhere in the course of time (see Supporting Information). One of the two Reviews in this issue highlights the most important works and achievements for electrochemical energy storage published in the last few years, including a critical analysis on the advantages, drawbacks, and future challenges of carbon and carbonbased materials for this purpose. The second Review describes recent promising activities and developments in heterogeneous catalysis, only using carbon and carbon nitride as catalysts, with a discussion on state-ofthe-art and future challenges for metal-free heterogeneous catalysis. The two Minireviews are devoted to the development of new synthetic pathways and processing routines towards nanostructured poly(benzimidazole) and their applications as proton conductors and catalysts, and to the production of carbonaceous materials from biomass or biomass-based 
products in a cheap, $\mathrm{CO}_{2}$-neutral, green, and sustainable manner with focus on the main directions and trends within this field. The Concept paper by Robert Schlögl brings arguments that the renewable energy supply systems require knowledge-based systemic chemical processes and optimized materials. The guest contribution by Gabriele Centi and Siglinda Perathoner from the University of Messina complements the line or arguments of this Special Issue with a critical discussion and comparison on the re- search advances on bioroutes, concentrated solar thermal and low temperature conversion using semiconductors and photoelectrocatalytic approach, in an attempt to define challenges and current limits, as well as to identify the priorities on which focus R\&D.

The guest editors thank all the colleagues who have contributed to this special issue, and the editorial office of ChemSusChem for assistance. 